\title{
Unknown Input Observers for Biological
}

\section{Processes}

\author{
A. Rapaport ${ }^{*, \star}$ D. Dochain ${ }^{* *, \star \star}$ J. Harmand ${ }^{* * *, \star}$ \\ G. Acuna ${ }^{* * *}$ \\ * UMR Analyse des Systèmes et Biométrie, INRA, 2 place Viala, \\ 34060 Montpellier, France \\ ** CESAME, Université catholique de Louvain, 4-6 avenue G. \\ Lemaître 1348 Louvain-la-Neuve, Belgium \\ *** Laboratoire de Biotechnologie de l'Environnement, INRA, Avenue \\ des étangs, 11100 Narbonne, France \\ **** Departamento de Ingenieria Informatica, Universidad de Santiago \\ de Chile, USACH, Av. Ecuador 3659 Santiago, Chile
}

\begin{abstract}
This paper addresses the question of estimating unknown inputs in biological processes. The unknown inputs are either constant or periodic (as e.g. in municipal wastewater treatment plants where the loading rate is unknown yet of a period equal to one day). In particular the designed observer is able to recover the unknown periodic inputs over a few periods.
\end{abstract}

Keywords: unknown input, periodic input, state observer, biological process.

\section{INTRODUCTION}

The monitoring of industrial biological processes is clearly an important issue due to the complexity of the involved processes, the lack of reliable and low cost sensors for the key process parameters and the uncertainty related to the dynamical models of such systems e.g. [2] [8]. Among the different challenges raised by the monitoring of the biological processes, one is specific to some classes of processes like municipal wastewater treatment plants (WWTP's) for which the organic load is typically unknown and time varying, yet with a typical periodicity of one day that follows the human domestic activities and the related production of wastewater and with a "nominal" periodic value which is roughly known. For industrial wastewater treatment plants, the organic load might also be unknown, yet varying whenever the type of production within the industrial plant is changed. In such instances, it is obvious that the main issue when operating wastewater treatment plant is disturbance rejection, and any tool that can provide reliable values of the changing organic load will be very useful to improve the performance of the WWTP.

The on-line estimation of systems with unknown inputs has a long history in research, starting from the seminal works of Kudva et al [13] and Fairman et al [9] (followed by the results of Darouach et al [5] [6]). In the context of chemical and biochemical systems, the asymptotic observers [2] [7], that do not require any knowledge of the process kinetics, belong to the class of state observers for systems with unknown inputs; yet they not handle

\footnotetext{
^ A. Rapaport and J. Harmand are with the INRA-INRIA team 'MERE', Montpellier, France.

${ }^{\star \star}$ Honorary Research Director FNRS, Belgium. E-mail : dochain@csam.ucl.ac.be, fax : +32-10-472180.
}

the specific issue of the unknown inputs, as mentioned above. However the asymptotic observers had been extended by considering cooperativity of the system to design interval observers that are able to reconstruct upper and lower bounds of the state by considering unknown but bounded inputs (like the loading rate) [12] [15]. The on-line estimation of unmeasured inputs in WWTP's had also been considered in [17] where an extended Luenberger observer design has been proposed. More recently, a modified asymptotic observer has been proposed to estimate the inlet glucose concentration from hydrogen and $\mathrm{CO}_{2}$ measurements in an anaerobic digestion process [1]. More generally, the issue of estimating systems with unknown inputs has been an active research area and new results (with e.g. results in active noise rejection) have been recently proposed [3] [4] [11]. Note that one of the main difficulty to design efficient tools for the on-line estimation of unknown inputs is linked to the causality principle, since the observer design has to rely on measurements that are indeed the effect or the result of the (unknown) cause, i.e. the unmeasured input.

In this paper we propose two state observers for unknown loading rates in biological processes, one when the unknown input is constant and the other one when it is periodic of known period. The design is typically that of a Luenberger observer where the unknown input is assimilated to an (unknown) state of the system. It is based on mass balance equations of the biological process. Stability and convergence results are provided for restrictive assumptions, and the performance of the observers are illustrated in numerical simulations. Proofs of convergence are based on Lyapunov transformations, that are recalled on the next section. 


\section{SYSTEM DESCRIPTION}

Consider the mass balance model of a stirred tank reactor (chemostat) involving a simple growth reaction

$$
\left\{\begin{array}{l}
\dot{X}=\mu(S) X-D(t) X \\
\dot{S}=-\frac{\mu(S) X}{Y}+D(t)\left(S_{\text {in }}-S\right)
\end{array}\right.
$$

where $X(\cdot)$ and $S(\cdot)$ hold for the biomass and substrate concentrations, respectively. $S_{i n}$ is the feed concentration. $D(\cdot), \mu(\cdot)$ and $Y$ are the dilution rate, the specific growth rate and the yield coefficient, respectively. $\mu(\cdot)$ is assumed to be a monotonic smooth non-negative function such that $\mu(0)=0$, with bounded derivative.

The model (1) has been extensively studied in the literature, under different hypotheses on the function $\mu(\cdot)$ and the dilution rate $D(\cdot)$ (see for instance [16]). The asymptotic behavior of the system (1) is related to the function $D(\cdot)$ and the values of $S_{i n}$ (for instance, values of $D$ larger than $\mu\left(S_{i n}\right)$ can lead to the washout of the biomass). This model is also used in biotechnology applications for the synthesis of stabilization laws or the estimation of unmeasured state variable. Nevertheless, in most studies, the input concentration $S_{\text {in }}$ is considered to be either perfectly known or an unknown input with known bounds. In this last case, controller and estimators are required to be robust with respect to the uncertainty on $S_{i n}$.

The aim of the present work is to propose estimators of $S_{i n}$ under reasonable assumptions. In a previous paper [14], an estimator for $S_{i n}$ has been designed and analyzed for the case when $S$ is measured. In the present instance, we would like to handle the more difficult situation when $\mathrm{X}(\mathrm{t})$ is available for on-line measurement, i.e. :

$$
y(t)=X(t)
$$

and propose two kinds of observers based on a priori knowledge of the variation of $S_{i n}$ :

(1) $S_{\text {in }}$ is constant,

(2) $S_{\text {in }}$ is periodic with known period.

Throughout the paper, we assume that the yield factor $Y$ is taken equal to 1 and that the manipulated variable $D(\cdot)$ is bounded, as well as its derivative, and fulfills a persistent excitation property

$$
D(t) \geq \underline{D}>0, \quad \forall t \geq 0 .
$$

With the state transformation

$$
M=X+S,
$$

system (1) can be equivalently rewritten

$$
\left\{\begin{array}{l}
\dot{X}=\mu(M-X) X-D(t) X \\
\dot{M}=D(t)\left(S_{\text {in }}-M\right)
\end{array}\right.
$$

Notice that when $D$ and $S_{i n}$ are constant, $S_{i n}$ is detectable: $S_{i n}=\lim _{t \rightarrow+\infty} X(t)+\mu^{-1}(D)$, which is no longer true when $D(\cdot)$ is time varying.

\section{LYAPUNOV TRANSFORMATIONS}

We recall the concept of Lyapunov transformation (see for instance [10]), that will be useful to analyze the convergence of the proposed estimators.

Consider a time-varying linear system

$$
\dot{x}(t)=A(t) x(t), \quad A(t) \in \mathcal{M}_{n \times n}(\mathbb{R}) .
$$

A $n \times n$ matrix $P(\cdot)$ defines a Lyapunov transformation when the following properties are fulfilled

(1) $P(\cdot)$ and $\dot{P}(\cdot)$ are continuous and bounded,

(2) $|\operatorname{det}(P(t))| \geq m>0$ for any $t \in[0,+\infty)$.

Then the system

$$
\dot{z}(t)=B(t) z(t)
$$

with

$$
B(t)=P^{-1}(t)(P(t) A(t) P(t)-\dot{P}(t))
$$

is said to be equivalent to (6).

It is straightforward to check that a Lyapunov transformation preserves state stability, because one has $x(t)=$ $P(t) z(t)$ for any $t \geq 0$. Then, a way to show the stability of the system (6) is to find a Lyapunov transformation $P(\cdot)$ such that proving the stability of the system $(7)$ is easy.

\section{OBSERVER WHEN $S_{I N}$ IS CONSTANT}

We assume here that the inlet substrate concentration $S_{\text {in }}$ is constant, and consider the observer

$$
\left\{\begin{array}{l}
\dot{\hat{X}}=[\mu(\hat{M}-y)-D(t)] y+G_{1}(t, y)(\hat{X}-y) \\
\dot{\hat{M}}=D(t)\left(\hat{S}_{i n}-\hat{M}\right)+G_{2}(t, y)(\hat{X}-y) \\
\dot{\hat{S}}_{i n}=G_{3}(t, y)(\hat{X}-y)
\end{array}\right.
$$

where $G_{i}(t, y)(i=1,2,3)$ are the state observer gains. Define the time function

$$
\delta(t)=\frac{\mu(\hat{M}(t)-y(t))-\mu(M(t)-y(t))}{\hat{M}(t)-M(t)} y(t) .
$$

Notice that $\delta(\cdot)$ stays uniformly bounded along any trajectory of (1)-(9), and is positive when $\mu(\cdot)$ is increasing.

Remark 1. When $\mu(S)=\bar{\mu} S$, where $\bar{\mu}$ is a positive constant, $\delta(t)$ is perfectly known: $\delta(t)=\bar{\mu} y(t)$. Otherwise, $\delta(t)$ can be approximated by $\hat{\delta}(t)=\mu^{\prime}(\hat{M}(t)-y(t)) y(t)$.

Proposition 2. When $\mu(\cdot)$ is linear, the gains

$$
\begin{aligned}
& G_{1}(t, y)=D(t)\left(1-\theta-\theta^{2}-\theta^{3}\right), \\
& G_{2}(t, y)=-D(t)^{2} \frac{1-\theta-\theta^{2}+\theta^{4}+\theta^{5}}{\delta(t)}, \\
& G_{3}(t, y)=-D(t)^{2} \frac{\theta^{6}}{\delta(t)},
\end{aligned}
$$

makes system (9) an exponential observer of $S_{i n}$, for large enough $\theta$. 
Proof. We first consider the time parameterization

$$
\tau(t)=\int_{0}^{t} D(s) d s \geq \underline{D} t, \quad t \geq 0 .
$$

Then the dynamics of the estimation error

$$
E=\left[\begin{array}{c}
\hat{X}-X \\
\hat{M}-M \\
\hat{S}_{i n}-S_{i n}
\end{array}\right]
$$

in time variable $\tau$ is given by

$$
\frac{d E(\tau)}{d \tau}=\underbrace{\left[\begin{array}{ccc}
1-\theta-\theta^{2}-\theta^{3} & \eta(\tau) & 0 \\
-\frac{1-\theta-\theta^{2}+\theta^{4}+\theta^{5}}{\eta(\tau)} & -1 & 1 \\
-\frac{\theta^{6}}{\eta(\tau)} & 0 & 0
\end{array}\right]}_{A_{\theta}(\tau)} E(\tau)
$$

where $\eta(\tau)=\delta(\tau) / D(\tau)$. One can easily check that

$$
S p\left(A_{\theta}(\tau)\right)=\left\{-\theta,-\theta^{2},-\theta^{3}\right\}, \quad \forall \tau \geq 0 .
$$

and that the time-varying matrix, when $\theta>1$,

$$
P_{\theta}(\tau)=\left[\begin{array}{ccc}
1 & 1 & 1 \\
-\frac{1-\theta^{2}-\theta^{3}}{\eta(\tau)} & -\frac{1-\theta-\theta^{3}}{\eta(\tau)} & -\frac{1-\theta-\theta^{2}}{\eta(\tau)} \\
\frac{\theta^{5}}{\eta(\tau)} & \frac{\theta^{4}}{\eta(\tau)} & \frac{\theta^{3}}{\eta(\tau)}
\end{array}\right]
$$

fulfills the property

$$
P_{\theta}^{-1}(\tau) A_{\theta}(\tau) P_{\theta}(\tau)=\Lambda_{\theta}=\left[\begin{array}{ccc}
-\theta & 0 & 0 \\
0 & -\theta^{2} & 0 \\
0 & 0 & -\theta^{3}
\end{array}\right] .
$$

Furthermore, $P_{\theta}(\cdot)$ fulfills the conditions given in Section 3 to be a Lyapunov transformation. Then, a straightforward computation gives the expression (8) of the matrix $B_{\theta}(\cdot)$ of the equivalent system.

$$
B_{\theta}(\tau)=\Lambda_{\theta}+\frac{\dot{\eta}(\tau)}{\eta(\tau)} \underbrace{Q_{\theta}\left[\begin{array}{lll}
0 & 0 & 0 \\
0 & 1 & 0 \\
0 & 0 & 1
\end{array}\right] Q_{\theta}^{-1}}_{C_{\theta}}
$$

with

$$
Q_{\theta}=\left[\begin{array}{ccc}
\theta^{3}-2 \theta^{4}+\theta^{5} & -1 & 0 \\
-\theta^{3}+2 \theta^{5}-\theta^{7} & 0 & -1 \\
\theta^{4}-\theta^{5}-\theta^{7}+\theta^{8} & 1 & 1
\end{array}\right]
$$

Consider the dynamics

$$
\frac{d Z(\tau)}{d \tau}=B_{\theta}(\tau) Z(\tau)
$$

and define the function $V(Z)=\frac{1}{2} Z^{t} Z$. Then one has, for $\theta>1$,

$$
\frac{d V(Z(\tau))}{d \tau} \leq 2\left(-\theta+\left|\frac{\dot{\eta}(\tau)}{\eta(\tau)}\right|\left\|C_{\theta}\right\|_{2}\right) V(Z(\tau))
$$

where $\left\|C_{\theta}\right\|_{2}=\sqrt{\lambda_{\max }\left(C_{\theta}^{t} C_{\theta}\right)}$. A straightforward but lengthy computation gives

$$
\left\|C_{\theta}\right\|_{2}=\sqrt{\frac{3 \theta^{6}+6 \theta^{5}+12 \theta^{4}+18 \theta^{3}+21 \theta^{2}+12 \theta+6}{\theta^{6}-2 \theta^{4}+\theta^{2}}}
$$

Notice that $\lim _{\theta \rightarrow+\infty}\left\|C_{\theta}\right\|_{2}=\sqrt{3}$, and $\dot{\eta}(\tau) / \eta(\tau)$ is uniformly bounded. Consequently, one has

$$
-\theta+\left|\frac{\dot{\eta}(\tau)}{\eta(\tau)}\right|\left\|C_{\theta}\right\|_{2} \leq-\nu<0, \quad \forall \tau \geq 0
$$

for large enough $\theta$. Finally, $V(\cdot)$ is a Lyapunov function for the dynamics (12), that guarantees the exponential convergence of $Z(\cdot)$. Under the time parameterization (11), one concludes that $E(\cdot)$ converges also exponentially toward the origin.

\section{OBSERVER WHEN $S_{I N}$ IS PERIODIC}

We assume here that the inlet substrate concentration $S_{i n}$ is periodic with known period $\omega$, provided by the following dynamical system :

$$
\left\{\begin{array}{l}
\dot{S}_{i n}=v \\
\dot{v}=-\omega^{2}\left(S_{i n}-a\right) \\
\dot{a}=0
\end{array}\right.
$$

where $a$ is the (unknown) mean value of $S_{i n}(\cdot)$. We therefore consider the following observer.

$$
\left\{\begin{array}{l}
\dot{\hat{X}}=[\mu(\hat{M}-y)-D(t)] y+G_{1}(t, y)(\hat{X}-y) \\
\dot{\hat{M}}=D(t)\left(\hat{S}_{i n}-\hat{M}\right)+G_{2}(t, y)(\hat{X}-y) \\
\dot{\hat{S}}_{i n}=\hat{v}+G_{3}(t, y)(\hat{X}-y) \\
\dot{\hat{v}}=-\omega^{2}\left(\hat{S}_{i n}-\hat{a}\right)+G_{4}(t, y)(\hat{X}-y) \\
\dot{\hat{a}}=G_{5}(t, y)(\hat{X}-y)
\end{array}\right.
$$

where $G_{i}(t, y)(\mathrm{i}=1$ to 5$)$ are the state observer gains.

Proposition 3. When $\mu(\cdot)$ is linear, the gains

$$
\begin{aligned}
& G_{1}(t, y)=D(t)+\sigma_{5}(\theta) \\
& G_{2}(t, y)=\frac{\omega^{2}-D(t)\left(D(t)+\sigma_{5}(\theta)\right)-\sigma_{4}(\theta)}{\delta(t)} \\
& G_{3}(t, y)=\frac{\sigma_{3}(\theta)-\omega^{2} \sigma_{5}(\theta)}{D(t) \delta(t)} \\
& G_{4}(t, y)=\frac{\omega^{2}\left(\sigma_{4}(\theta)-\omega^{2}\right)-\sigma_{2}(\theta)}{D(t) \delta(t)} \\
& G_{5}(t, y)=\frac{\sigma_{1}(\theta)}{\omega^{2} D(t) \delta(t)}
\end{aligned}
$$

where $\delta(\cdot)$ is defined in (10) and

$$
\begin{aligned}
& \sigma_{5}(\theta)=-\theta-\theta^{2}-\theta^{3}-\theta^{4}-\theta^{5} \\
& \sigma_{4}(\theta)=\theta^{3}+\theta^{4}+2 \theta^{5}+2 \theta^{6}+2 \theta^{7}+\theta^{8}+\theta^{9} \\
& \sigma_{3}(\theta)=-\theta^{6}-\theta^{7}-2 \theta^{8}-2 \theta^{9}-2 \theta^{10}-\theta^{11}-\theta^{12} \\
& \sigma_{2}(\theta)=\theta^{10}+\theta^{11}+\theta^{12}+\theta^{13}+\theta^{14} \\
& \sigma_{1}(\theta)=-\theta^{15}
\end{aligned}
$$

makes system (13) an exponential observer of system $S_{i n}(\cdot)$ for large enough $\theta$. 
Proof. The dynamics of the estimation error

$$
E=\left[\begin{array}{c}
\hat{X}-X \\
\hat{M}-M \\
\hat{S}_{i n}-S_{i n} \\
\hat{v}-v \\
\hat{a}-a
\end{array}\right]
$$

is solution of the linear time-varying system

$$
\dot{E}(t)=\underbrace{\left[\begin{array}{ccccc}
G_{1}(t, y) & \delta(t) & 0 & 0 & 0 \\
G_{2}(t, y) & -D(t) & D(t) & 0 & 0 \\
G_{3}(t, y) & 0 & 0 & 1 & 0 \\
G_{4}(t, y) & 0 & -\omega^{2} & 0 & \omega^{2} \\
G_{5}(t, y) & 0 & 0 & 0 & 0 e
\end{array}\right]}_{A_{\theta}(t)} E(t)
$$

One can easily check that the gains $G_{i}(\cdot)$ have been determined such that

$$
S p\left(A_{\theta}(t)\right)=\left\{-\theta,-\theta^{2},-\theta^{3},-\theta^{4},-\theta^{5}\right\}, \quad \forall t \geq 0
$$

With the help of a symbolic computation software, one can find a matrix $P_{\theta}(t)$ such that

$$
P_{\theta}^{-1}(t) A_{\theta}(t) P_{\theta}(t)=\underbrace{\left[\begin{array}{ccccc}
-\theta & 0 & 0 & 0 & 0 \\
0 & -\theta^{2} & 0 & 0 & 0 \\
0 & 0 & -\theta^{3} & 0 & 0 \\
0 & 0 & 0 & -\theta^{4} & 0 \\
0 & 0 & 0 & 0 & -\theta^{5}
\end{array}\right]}_{\Lambda_{\theta}} .
$$

For $\theta \neq 1$, an instance of such $P_{\theta}(t)$ is given by the expression below

$$
\left[\begin{array}{ccccc}
\rho & \theta \rho & \theta^{2} \rho & \theta^{3} \rho & \theta^{4} \rho \\
\alpha_{2345} & \theta \alpha_{1345} & \theta^{2} \alpha_{1245} & \theta^{3} \alpha_{1235} & \theta^{4} \alpha_{1234} \\
\beta_{567789} & \theta \beta_{456789} & \theta^{2} \beta_{356679} & \theta^{3} \beta_{345678} & \theta^{4} \beta_{345567} \\
\gamma_{123789,10} & \gamma_{234789,11} & \theta \gamma_{134679,10} & \theta^{2} \gamma_{124578,9} & \theta^{3} \gamma_{123567,8} \\
\theta^{14} & \theta^{14} & \theta^{14} & \theta^{14} & \theta^{14}
\end{array}\right]
$$

where

$$
\begin{aligned}
& \rho=\omega^{2} D(t) \delta(t), \\
& \alpha_{i j k l}=\omega^{2} D(t)\left(\zeta_{i j k l}-D(t)\right), \\
& \beta_{i j k l m n}=\omega^{2}\left(\zeta_{i j k l m n}-\omega^{2}\right), \\
& \gamma_{i j k l m n, o}=\omega^{2} \theta^{2}\left(\zeta_{l m n o}-\omega^{2} \zeta_{i j k}-\omega^{2}\right),
\end{aligned}
$$

When $\theta>1, P_{\theta}(\cdot)$ is a Lyapunov transformation, which leads to an equivalent system $\dot{Z}=B_{\theta}(t) Z$ with

$$
B_{\theta}(t)=\Lambda_{\theta}-P_{\theta}^{-1}(t) \dot{P}_{\theta}(t) .
$$

Notice that one can write

$$
\dot{P}_{\theta}(t)=\frac{\dot{\delta}(t)}{\delta(t)} R P_{\theta}(t)+\frac{\dot{D}(t)}{D(t)}\left[S-\frac{D(t)}{\delta(t)} T\right] P_{\theta}(t)
$$

with

$R=\left[\begin{array}{lllll}1 & 0 & 0 & 0 & 0 \\ 0 & 0 & 0 & 0 & 0 \\ 0 & 0 & 0 & 0 & 0 \\ 0 & 0 & 0 & 0 & 0 \\ 0 & 0 & 0 & 0 & 0\end{array}\right], S=\left[\begin{array}{lllll}1 & 0 & 0 & 0 & 0 \\ 0 & 1 & 0 & 0 & 0 \\ 0 & 0 & 0 & 0 & 0 \\ 0 & 0 & 0 & 0 & 0 \\ 0 & 0 & 0 & 0 & 0\end{array}\right], T=\left[\begin{array}{lllll}0 & 0 & 0 & 0 & 0 \\ 1 & 0 & 0 & 0 & 0 \\ 0 & 0 & 0 & 0 & 0 \\ 0 & 0 & 0 & 0 & 0 \\ 0 & 0 & 0 & 0 & 0\end{array}\right]$.

One can also easily check, with the help of a symbolic computation software, that the following properties are fulfilled

$$
\begin{aligned}
& \lim _{\theta \rightarrow+\infty} P_{\theta}^{-1}(t) R P_{\theta}(t)=\left[\begin{array}{llllr}
0 & 0 & 0 & 0 & 0 \\
0 & 0 & 0 & 0 & 0 \\
0 & 0 & 0 & 0 & 0 \\
0 & 0 & 0 & 0 & -1 \\
0 & 0 & 0 & 0 & 1
\end{array}\right], \\
& \lim _{\theta \rightarrow+\infty} P_{\theta}^{-1}(t) S P_{\theta}(t)=\left[\begin{array}{rrrrr}
0 & 0 & 0 & 0 & 0 \\
0 & 0 & 0 & 0 & 0 \\
0 & 0 & 0 & -1 & -1 \\
0 & 0 & 0 & 1 & 0 \\
0 & 0 & 0 & 0 & 1
\end{array}\right], \\
& \lim _{\theta \rightarrow+\infty} P_{\theta}^{-1}(t) T P_{\theta}(t)=0
\end{aligned}
$$

Let $V(Z)=\frac{1}{2} Z^{t} Z$. From (14), one can write

$$
\dot{V} \leq 2\left(-\theta+\left\|P_{\theta}^{-1}(t) \dot{P}_{\theta}^{-1}(t)\right\|_{2}\right) V
$$

when $\theta>1$. Then, from (15), (16), (17), (18), $\dot{\delta} / \delta$ and $\dot{D} / D$ being bounded, one obtains

$$
-\theta+\left\|P_{\theta}^{-1}(t) \dot{P}_{\theta}^{-1}(t)\right\|_{2} \leq-\nu<0, \quad \forall t \geq 0
$$

for large enough $\theta$. Finally $V(\cdot)$ is a Lyapunov function that guarantees the exponentially convergence of $Z(\cdot)$, or equivalently $E(\cdot)$, toward 0 .

\section{SIMULATIONS}

Simulations have been conducted with a linear growth function $\mu(\cdot)$ and fluctuating dilution rate $D(\cdot)$. Tuning parameter $\theta$ has been chosen equal to 1.5 and measurement $y(\cdot)$ has been corrupted by $2 \%$ white noise.

Trajectories are depicted on Figures 1 and 2, for piecewise constant and piecewise periodic $S_{i n}$, respectively.

\section{CONCLUSION}

For biological processes with linear growth functions, exponential observers for unknown input concentration have been proposed, for the cases of constant unknown input, or periodic unknown input, with known period. Proofs of convergence are based on the use of Lyapunov transformations.

Simulations with non-linear $\mu(\cdot)$ and approximation of $\delta(\cdot)$ are not presented here. Robustness with respect to on uncertainty on $\delta(\cdot)$ will be matter of a future work.

\section{REFERENCES}

[1] Aceves-Lara C.-A., E. Latrille and J.P. Steyer (2007). Input and states estimations of biohydrogen production. Proc. 10th IFAC Symp. Computer Applications on Biotechnology, 93-98.

[2] Bastin G. and D. Dochain (1990). On-line Estimation and Adaptive Control of Bioreactors. Elsevier, Amsterdam.

[3] Guo X. and M. Bodson (2007). Adaptive rejection of multiple sinusoids of unknown frequency. Proc. ECC'0\%, 121-128.

[4] Boulkroune B., M. Darouach and M. Zasadzinski (2007). Moving horizon estimation for discrete time linear systems with unknown inputs. Proc. ECC'07, 2875-2878. 
Sin and its estimator

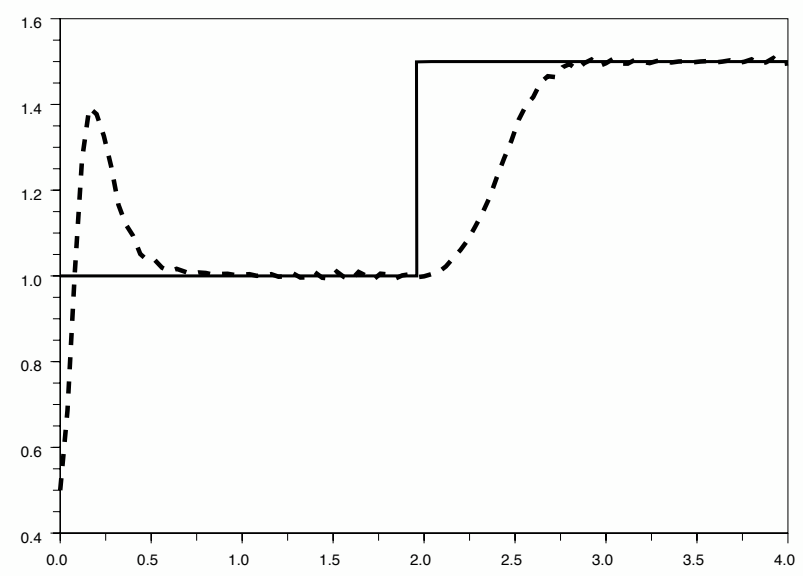

\section{$\mathrm{y}$ and estimator of $\mathrm{X}$}

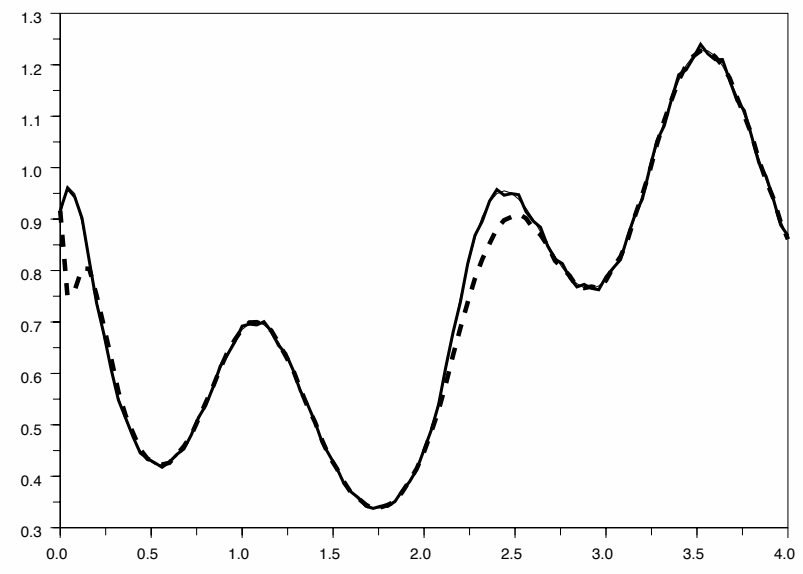

Fig. 1. Simulation with piecewise constant $S_{i n}$.

[5] Darouach M. (1994). On the novel approach to the design of the unknown input observers. IEEE Trans. Aut. Control, 39(3), 698-699.

[6] Darouach M., M. Zasadzinski and M. Hayar (1996). Reduced-order observer design for descriptor systems with unknown inputs. IEEE Trans. Aut. Control, 41(7), 1068-1072.

[7] Dochain D., M. Perrier and B.E. Ydstie (1992). Asymptotic observers for stirred tank reactors. Chem. Eng. Sci., 47, 4167-4178.

[8] D. Dochain (2003). State and parameter estimation in chemical and biochemical processes : a tutorial. Journal of Process Control, 13, 801-818.

[9] Fairman F.W., S.S. Mahil and L.Luk (1984). Disturbance decoupled observer design via singular value decomposition. IEEE Trans. Aut. Control, AC-29 (1), 84-86.

[10] Gantmacher F.R. (1959). The theory of matrices, Chelsea, New-York.

[11] Gillijns S., N. Haverbeke and B. De Moor (2007). Information, covariance and square-root filtering in the presence of unknown inputs. Proc. $E C C^{\prime} 0^{\circ}$, 2213-2217.
Sin and its estimator

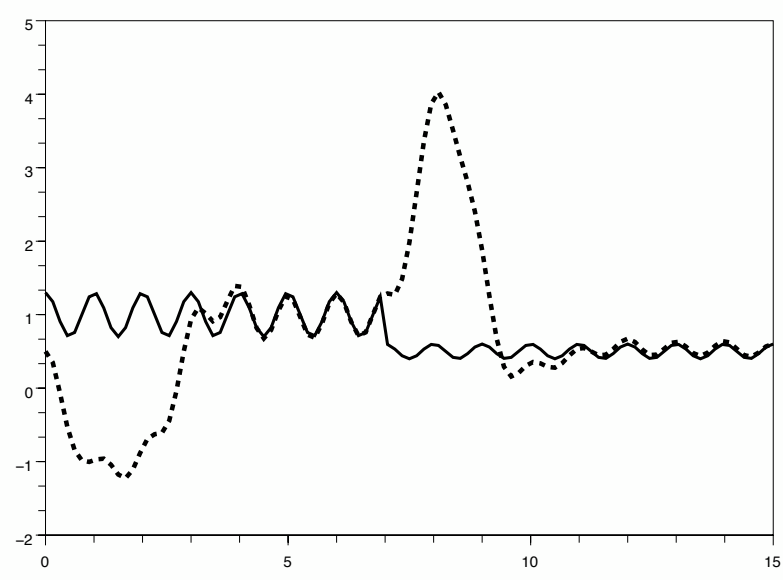

$\mathrm{y}$ and estimator of $\mathrm{X}$

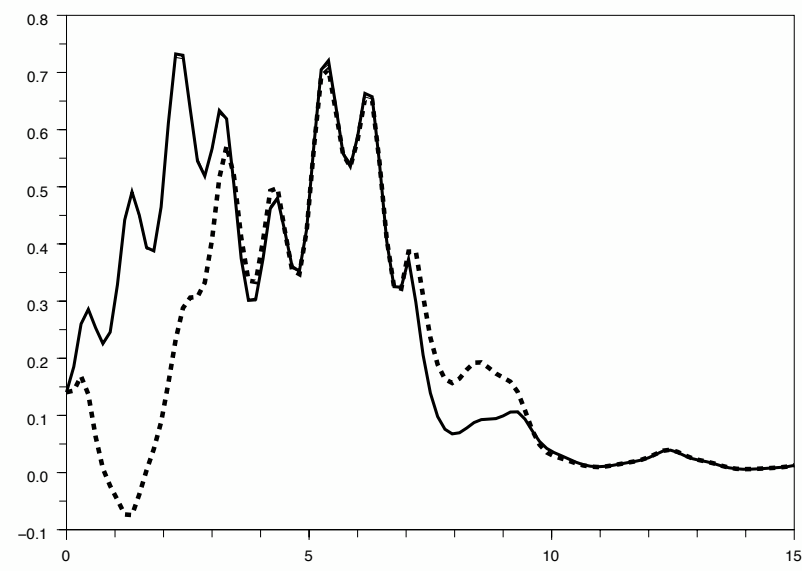

Fig. 2. Simulation with piecewise periodic $S_{i n}$.

[12] Gouzé J.L., A. Rapaport and M.Z. Hadj-Sadok (1999). Interval observers for uncertain biological systems. J. Ecological Modelling, 133 (1-2), 45-56.

[13] Kudva P., N. Viswanadham and A. Ramakrishna (1980). Observers for linear systems with unknown inputs. IEEE Trans. Aut. Control, AC-25 (1), 113-115.

[14] Rapaport A., F. Mazenc and J. Harmand (2006). Output tracking of bioprocesses through recirculation with unknown input concentration. Proc. ADCHEM'06, .

[15] Rapaport A. and D. Dochain (2003). Interval observers for uncertain processes. Mathematical Biosciences, 193 (2), 235-253.

[16] Smith H.L and P. Waltman (1995). The Theory of the Chemostat. Dynamics of Microbial Competition. Cambridge University Press, Cambridge.

[17] Theilliol D., J.C. Ponsart, J. Harmand, C. Join and P. Gras (2003). On-line estimation of unmeasured inputs for anaerobic wastewater treatment processes. Control Engineering Practice, 11, 10071019. 